\title{
eXiTCDSS: A framework for a workflow-based CBR for interventional Clinical Decision Support Systems and its application to TAVI
}

\author{
Andres El-Fakdi ${ }^{\mathrm{a}}$, Francisco Gamero ${ }^{\mathrm{a}}$, Joaquim Meléndez ${ }^{\mathrm{a}}$, Vincent \\ Auffret $^{\text {b,c,d }}$, Pascal Haigron ${ }^{c, d}$ \\ ${ }^{a}$ Control Engineering and Intelligent Systems Group eXiT, University of Girona \\ Campus Montilivi Building PIV, 17071 Girona, Spain. \\ ${ }^{b}$ Division of Cardiology, Pontchaillou University Hospital \\ Rennes, F-35000, France. \\ ${ }^{c}$ Université de Rennes 1, LTSI \\ Rennes, F-35000, France. \\ ${ }^{d}$ INSERM, U1099 \\ Rennes, F-35000, France.
}

\begin{abstract}
Clinical Decision Support Systems (CDSSs) should form an important part of the field of clinical knowledge management technologies through their capacity to support the clinical process and use of knowledge, including knowledge maintenance and continuous learning, from diagnosis and investigation through surgery, treatment and long-term care. The work presented shows a workflow-based CDSS designed to give case-specific assessment to clinicians during complex surgery or Minimally Invasive Surgerys (MISs). Following a perioperative workflow, the designed software will use a Case-Based Reasoning (CBR) methodology to retrieve similar past cases from a case base to provide support at any particular point of the process. The graphical user interface allows easy navigation through the whole support progress, from the initial configuration steps to the final results organized as sets of experiments easily visualized in a user-friendly way. The eXiTCDSS tool is presented giving support to a recent complex minimally invasive surgery
\end{abstract}

Email addresses: andres.elfakdi@udg.edu (Andres El-Fakdi), paco.gamero@udg.edu (Francisco Gamero), joaquim.melendez@udg.edu (Joaquim Meléndez), v. auffret@gmail.com (Vincent Auffret),

Pascal. Haigron@univ-rennes1.fr (Pascal Haigron) 
which is receiving growing attention lately, the Transcatheter Aortic Valve Implantation (TAVI). The results obtained are presented on a basis of a real TAVI case base of 82 patients operated at Rennes University Hospital.

Keywords: Clinical Decision Support Systems, Case-Based Reasoning, Clinical workflows, Transcatheter Aortic Valve Implantation.

\section{Introduction}

Nowadays, medicine and health fields are getting more and more involved with computer science. Among all branches, the main focus of the current research points towards Artificial Intelligence (AI) to improve the performance of Clinical Decision Support Systems (CDSSs). In a general term, CDSSs comprise a large spectrum of systems which provide clinicians, staff, patients, and other individuals with knowledge and person-specific information, intelligently filtered and presented at appropriate times, to enhance health and health care (Berner, 2009). Although the first attempts to supply health services with computerized systems appeared in late 1950s (Ledley \& Lusted, 1959), CDSSs have been used in clinical practice since 1970 with the appearance of the first clinical advisory systems (Dombal et al., 1972; Shortliffe, 1976; Warner, 1979). Since then, several CDSSs based on AI algorithms have been designed and are widely used in hospitals and medical centers, shifting from merely administrative systems to the actual CDSSs and they are proved to be very useful not only to help the clinical staff in making decision but for patients also.

CDSSs should form an important part of the field of clinical knowledge management technologies through their capacity to support the clinical process and use of knowledge,including knowledge maintenance and continuous learning, from diagnosis and investigation through surgery, treatment and long-term care. Arguments for and against the value of CDSSs have been discussed over the years. Among its potential benefits, as stated in (Coiera, October 2003), CDSSs could improve patient safety through reduced medication errors and adverse events. Also, they should improve quality of care by increasing pathways, guidelines and documentation available for patients. Another advantage is that CDSSs may improve efficiency in health care delivery, reducing costs through faster order processing and avoiding test duplication. As drawbacks, clinicians may see CDSSs as a threat to clinical judgment and sometimes too inflexible, with difficulties to depart from or- 
dered, pre-prepared paths. Also, computer-supported decision systems promote over-reliance on software decisions which may limit clinicians' freedom to think at some point. In the same way, bad designed systems can create extra work or extend clinical procedures more than necessary. Finally, maintenance costs, professional support and training needed by the medical staff in order to use the software properly could also be seen as arguments against the utilization of CDSSs. Despite several successes, their impact on routine clinical practice has not been as strong as expected, specially due to the barriers to their implementation, which still remain in place. Therefore, up to the date, CDSSs have not been largely tested and, given their fast evolution and the limited range of clinical settings in which they have been used, it is mandatory that CDSSs should be rigorously evaluated before widespread introduction into clinical practice (Evans et al., 1998).

Decision support can be provided at various stages in the care process, from preventive care through diagnosis and treatment to monitoring and follow-up. As detailed in Perreault \& Metzger (1999), computer CDSSs can be designed to support four basic clinical functions. First, giving administrative support, aiding in clinical coding and documentation, authorization of procedures, and referrals. Second, managing clinical complexity, keeping patients on research and chemotherapy protocols, tracking orders, referrals follow-up, and preventive care. The third function deals with cost control, programming CDSSs to monitor medication orders with the objective of avoiding duplicate or unnecessary tests. A fourth stage of application, which represents the focus of this work, involves CDSSs with low level decision support, helping in clinical diagnosis and treatment plan processes, giving case-specific support in highly complex surgery operations as in Minimally Invasive Surgerys (MISs), promoting use of best practices and guidelines based on population case management.

CDSSs are classified into two main groups, depending on whether they are knowledge-based systems, or non knowledge-based systems (Berner, 2009). The knowledge-based CDSSs are the most common type of CDSSs used in clinics and hospitals. Many of today's knowledge-based CDSSs arose out of earlier expert systems research, where the aim was to build a computer program that could simulate human thinking (Shortliffe et al., 1973; Miller et al., 1982). They are structured around rules mostly in the form of IF-THEN statements. Most of knowledge-based systems consist of three parts, the knowledge base, inference engine, and mechanism to communicate (Wyatt \& Spiegelhalter, 1991). The rules are associated with compiled data extracted 
from a knowledge base. The inference engine combines the rules from the knowledge base with the patient's data. The communication mechanism will allow the system to show the results to the user as well as have input into the system. Non knowledge-based CDSSs use AI through machine learning techniques, which allows the computer to learn from past experiences and to recognize patterns in the clinical data (Marakas, 1999). Artificial Neural Network (ANN) (Baxt, 1995; Holst et al., 2000; Olsson et al., 2002) and genetic algorithms (Laurikkala et al., 1999) are two common types of non knowledgebased systems. The fusion of a knowledge base with non knowledge-based machine learning techniques results into an hybrid system. Hybrid systems extract the best from both methodologies, finally resulting into an overall improvement of the system performance and thus providing an optimal solution for clinical decision support systems (Demmer-Fushman \& Lin, 2007). This paper will focus on this kind of hybrid systems, presenting a software framework for CDSS which uses a well-known lazy learning technique called Case-Based Reasoning (CBR) (Aamodt \& Plaza, 1994) and a past patients case base to asses clinicians. Although rule-based approaches can be used to implement CDSS, rules for these systems would become very complex and difficult to compile (Wills \& Watson, 2004). Storing experience lends itself well to a case-based approach, whereas encoding experience knowledge in rules is not as intuitive. In fact cases can also be seen as rules (one case one rule) specific for each case. CBR systems also automatically adapt with experience, while rule-based approaches may need more user-interaction and understanding.

From a software oriented point of view, a CBR-based CDSSs is designed to directly aid in clinical decision making in which characteristics of individual patients are matched to a computerized knowledge base for the purpose of generating patient-specific assessments or recommendations that are then presented to clinicians for consideration (Hunt et al., 1998). CBR is the process of solving new problems based on the solutions of similar past problems. In the clinical field, CBR has been specifically used in successful CDSSs. An integration of CBR and rule-based reasoning was used in systems for the planning of ongoing care of Alzheimer's patients (Marling \& Whitehouse, 2001) and for the management of Diabetes patients (Bellazi et al., 1999).

The eXiTCDSS tool is presented giving support to a recent complex minimally invasive surgery which is receiving growing attention lately, the Transcatheter Aortic Valve Implantation (TAVI) (Webb \& Cribier, 2011). In TAVI, a synthetic valve is transported to the heart through a small hole 
made in groin. This procedure can be compared to that performed when placing a stent, or performing balloon angioplasty. Nowadays, there are two current market leaders whose devices have earned CE Mark approval in Europe and are available to physicians for TAVI in appropriate patients; the CoreValve device (a self-expanding valve prosthesis consisting of a Nickeltitanium frame with a tri-leaflet valve fashioned out of porcine pericardium mounted within) and the SAPIEN device by Edwards Lifesciences (a balloonexpandable tubular metal stent with a tri-leaflet valve fashioned out of bovine pericardium mounted within). This technique was first developed in Europe, where it was initially performed in 2002. Since then, more than 10000 patients have benefited from it and the results have shown the procedure to be effective in improving functioning in the patients with severe aortic stenosis. In the recent years TAVI is assuming a major role in the routine management of patients with aortic stenosis and now TAVI is considered the standard in patients who are not candidates for conventional surgical Aortic Valve Replacement (AVR). On the basis of almost 10 years of experience TAVI also appears to be a reasonable option for some operable, but high-risk patients. Nevertheless considerable work needs to be done before TAVI is expanded into lower risk groups.

This work presents a workflow-based CDSS designed to give case-specific assessment to clinicians during complex surgery. For this purpose, a real TAVI case base of 82 patients operated at Rennes University Hospital is used as case base. The article is structured as follows. In Section 2 a description of the workflow management during an intervention is detailed. Also, recent applications of CDSS for surgical processes are reviewed. Section 3 introduces the eXiTCDSS framework. Also, this section describes the components and functionalities of the application. In Section 4, the TAVI MIS is described. The application of eXiTCDSS is applied to the TAVI procedure. In Section 5, experimental results with a real TAVI patient case base are presented. Finally, conclusions and future work are included in Section 6 .

\section{CDSS Integration with Clinical Workflow}

Recent introduction of new clinical techniques such as MIS has led to several technological innovations inside the Operation Room (OR). All these advances, however, create new difficulties, such as inadequate information transparency, limited access, and poor visualization. Therefore, clinicians must rely on advancements in medical imaging technology (Dugas et al., 


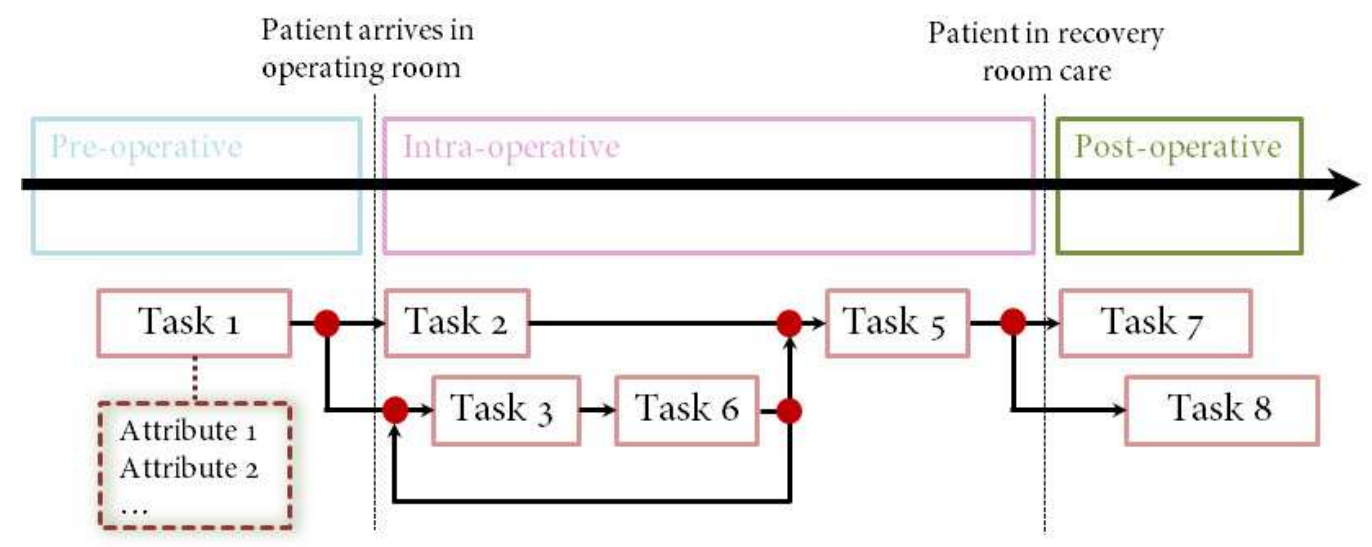

Figure 1: Schematic workflow showing an exemplified operative process. The process is divided into phases, each one containing subtasks. These tasks are filled with attributes.

2002). These limitations in MIS are constantly giving rise to new research and development in the area of CDSSs. Such systems are providing realtime image guidance and task automation support while the clinician is performing the intra-operative tasks (Wood et al., 2007).

Empirical studies demonstrate the benefits of including decision support into complex workspace scenarios, which leads to safer working environments and prevention of errors (Durieux et al., 2000). Examples of successful applications of CDSSs into clinical workflows comprise computer based patient record systems (Patel et al., 2000), knowledge management systems for biomedical engineering (Rinkus et al., 2004) and computer based training systems in pathology (Crowley et al., 2003). Although multiple factors are believed to affect the success or failure of CDSS intervention implementation, a helpful CDSS is dependent on the completeness and accuracy of the evidence base used to support it. Also, from the successful applications mentioned before it can be extracted that integration with workflow is key to success. How to integrate the CDSS with clinician workflow, however, remains a challenge, in part because there are no current standards for clinical workflow (Das \& Eichner, 2010).

Over the past few years, significant research in the area of medical informatics points to the importance of understanding workflow processes to support the development of CDSSs for complex workspaces (Sittig et al., 2008; Patel et al., 2001). Nowadays there is a demand from the clinicians to extend the capabilities of modern CDSSs from a pure consult database 
towards a software system fully integrated with hospital routines and procedures, inside and outside the OR. As demand for CDSSs tools increases, there is need to disseminate these support systems in a way that incorporates information about important environmental factors like the workflow.

From the health care point of view, the clinical workflow describes how the patient and the flow of clinical information interact with clinical providers in the practice, affecting the efficiency of care delivery, patient satisfaction, and the quality of the clinician's time. Although there is no universally agreed upon definition of the term workflow, for the purpose of this article, we have taken the workflow definition stated in Carayon et al. (2010); Niazkhani et al. (2009); Unertl et al. (2010) which defines a clinical workflow as a modular sequence of tasks, with a distinct beginning and end, performed for the specific purpose of delivering clinical care. In order to implement a workflow-based CDSS, tasks, timing and involved subprocesses must by identified first. Therefore, the proposed workflow has been specified at up to four levels of detail: 1) clinical workflow, 2) phase, 3) task, and 4) attribute. Figure 1 shows a schematic workflow of an exemplified operative process where the previously mentioned levels have been illustrated. The first level of the workflow represents the particular workflow itself. The second level describes the phases, being a phase the primordial division of the specific clinical workflow. For the particular example shown in Figure 1, each phase corresponds to the pre, intra, and post-operative periods. In the same way, every phase has been split into tasks, a task being any particular step taken during each phase e.g. apply anesthesia, initial puncture location or valve final placement. Each task has a different number of distinguishable items or attributes associated. These attributes refer to all the important values or considerations that the medical staff will take into account during the resolution of a task. The attributes can be described as numerical data, text data, categorical data, and boolean data. As numerical data it can be considered blood count, coagulation parameters, age, size, or specific physiologic measurements. The text data comprises those textual items regarding the patient's pathological or surgical history as well as possible allergies. The categorical, in fact ordered categorical data, comprises attributes which measure a certain degree of intensity, e. g. amount of calcification or valve regurgitation while the boolean data confirms or denies the presence of an attribute, for example the vascular tortuosity or the existence of coronary flow damage. During the intervention execution and according to the current information being generated, the CDSS has to be capable to identify the 


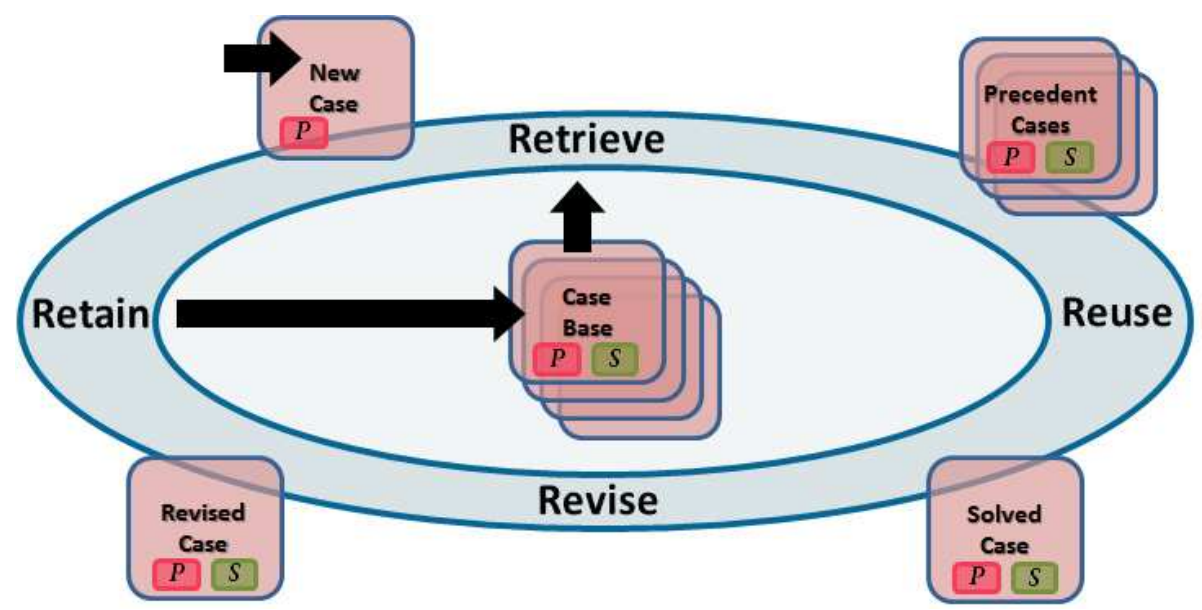

Figure 2: Four-step process of Case-Based Reasoning.

phase, the task, and the attributes involved. Then, the software will use the CBR engine to retrieve the most similar cases to the current one together with the solutions that were adopted. The framework eXiTCDSS presented in this article provides the required tools to define a case structure for any clinical procedure based on a workflow. With a case base of indexed past interventions organized as cases, once a new patient case arrives, clinicians are able to on-line retrieve any case similar to the new one, with its correspondent tasks and associated attributes at any point along the workflow. By accessing this information, the medical staff will have more information which may help them to select the next action properly.

\section{The eXiTCDSS Framework}

Case-Based Reasoning (CBR) is a technique of artificial intelligence that attempts to solve a given problem within a specific domain by adapting established solutions to similar problems (Aamodt \& Plaza, 1994). CBR has been formalized for purposes of reasoning and learning based on the exploitation of existing similar historical records as humans do. It has been argued that CBR is not only a powerful method for computer reasoning, but also a pervasive behavior in everyday human problem-solving; or, more radically, that all reasoning is based on past cases personally experienced. These features make CBR a good contender for any decision support system (Watson, 2002). 


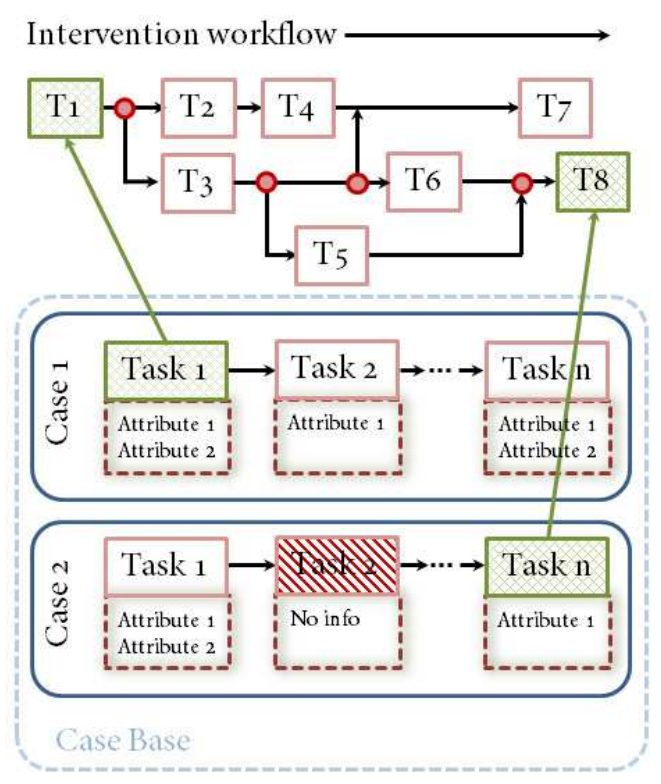

Figure 3: Retrieval process along the operative workflow. At any task step as the procedure advances similar cases to the current case are retrieved. Green arrows depict a similarity found while in a particular task between the current case and a case from the case base.

As can be seen in Figure 2, four main phases of action are defined in the CBR methodology: retrieve, reuse, revise and retain. For example, in TAVI, a case base contains information about patients that have been operated in the past. Using this case base, a CBR system is able to give advice to future TAVI cases by following the four phases: retrieve, reuse, revise and retain. First, in the retrieve phase, the current case is compared with all the past experiences in the case base, and the most similar are recovered. Given a target problem, during the retrieve step, cases from memory that are relevant to solving it are recovered. A case is a whole although usually two parts can be identified: the problem and the solution. The problem incorporates specific case information which characterizes the case whereas the solution incorporates information for solving any particular problem. Next, in the reuse phase, a solution to the current case is determined based on the solutions found in the retrieved cases, which are mapped to target the actual problem. This may involve adapting the solution as needed to fit the new situation. Third, the computed solution is evaluated in the revise phase. Finally, the retain phase analyzes whether to retain the case in the case base.

In order to design a case-based reasoning system adapted to an operation 
workflow like the one described in Section 2, we should determine a case model, case indexing and a similarity metric. As illustrated in Figure 3, every case of the case base is defined as a set of attributes classified among the different tasks which compound the whole operative workflow. The stored cases do not need to contain the same number of attributes, as it will depend on the amount of information available when storing each case. Therefore, some cases will be richly filled with useful information concerning all the tasks of the workflow while others can be poorly defined with missing information in certain tasks or even non-existent tasks at all, as not all of them are mandatory and depend on each patient.

When the intervention begins, parallel to the workflow progression described in Figure 3, the CBR mechanism retrieves specific and contextual problem information of similar past cases. Such assessment step determines which cases address problems most similar to the current problem, to identify them as starting points for solving the new problem. Once the operation ends, clinicians proceed with the new case revision, which evaluates and adjusts the adapted solutions and, if suitable, the new case will be retained with the system learning from the situation by storing the result as a new case for future use. The software has been designed to work either on-line inside the OR or off-line, as a knowledge database for patient study.

As the eXiTCDSS framework goes beyond pure CBR prototyping and aims to support workflow-oriented decision support, other elements are required in addition to the basic CBR modules. In order to manipulate the data, a common representation of cases is required. Also, three main components are distinguished: the workflow editor, the results navigator and the CBR engine.

\subsection{Case representation}

eXiTCDSS requires a plain Coma Separated Value (CSV) file to handle the data. Figure 4 shows an example fragment of a CSV file used by the eXiTCDSS application. As can be seen in the figure, the first four rows of the file conform the header, which contains information used to characterize every attribute. Each column represents an attribute. Thus, the first row of the header details the attribute description that is used to identify the attribute by the user (for example, "Annular calcification"). The second row of the header corresponds to the attribute identification used by the architecture to refer to it (it is usually similar as the description but in a compressed form, as for example, "Annularcalcification"). The third row of the header contains 


\begin{tabular}{c|c|c|c|c|c}
$\begin{array}{c}\text { Attribute Name } \\
\text { Attribute id }\end{array}$ & $\begin{array}{c}\text { Gender } \\
\text { gender }\end{array}$ & $\begin{array}{c}\text { Age } \\
\text { age }\end{array}$ & $\begin{array}{c}\text { Size } \\
\text { size }\end{array}$ & $\begin{array}{c}\text { Weight } \\
\text { weight }\end{array}$ & $\begin{array}{c}\text { Ejection Fraction } \\
\text { ejectionfraction }\end{array}$ \\
$\begin{array}{c}\text { Attibute type } \\
\text { Attribute weight }\end{array}$ & 0 & 1 & 1 & 1 & 1 \\
\hline \hline 0 & 1 & 1 & 1 & 1 & 1 \\
\hline 1 & F & 88 & 165 & 67 & 20 \\
\hline 2 & M & 75 & 170 & 81 & 55 \\
\hline 3 & F & 87 & 155 & 75 & 55 \\
\hline 4 & F & 77 & 158 & 90 & 60 \\
\hline 5 & M & 82 & 174 & 92 & 55 \\
\hline
\end{tabular}

Figure 4: CSV file where data is extracted from. The header contains attribute description, identification, data type and attribute weighting. Below, each row corresponds to a case and the columns describe the attributes.

the attribute type that is used by the system to assign the right operators for every attribute type (-1 ignore, 0 boolean, 1 numerical, 2 textual, 3 categorical). Finally, the fourth row of the header corresponds to the attribute weighting. As it will be detailed in a further section, the weighting allows the user to promote particular attributes by increasing its weight in comparison to others and, therefore, giving more importance to certain aspects of a patient profile which the user consider crucial to find the best solution to a particular problem. After the header comes the patient case base. Each row below the header corresponds to a case of the case base. This representation covers most of the data used in medical applications (Microsoft excel or Statistical Package for the Social Sciences (SPSS) files) and is easy to manage and general enough to be used by any of the current CBR techniques, mainly distance functions.

\subsection{The workflow editor}

To support a workflow-oriented CDSS software, a workflow editor tool has been developed inside the main application so that users can define a workflow file. As illustrated in Figure 5, all the needed features to describe any type of workflow are displayed in a window independent interface that can be accessed from the tools label in the top area of the main window frame of the platform. This functionality includes a shapes menu with all the types of boxes, containers and arrows used to define the workflow structure with all the needed phases and tasks. The created figures are editable and colorable, with a label for its identification. After the structure definition, on the right 


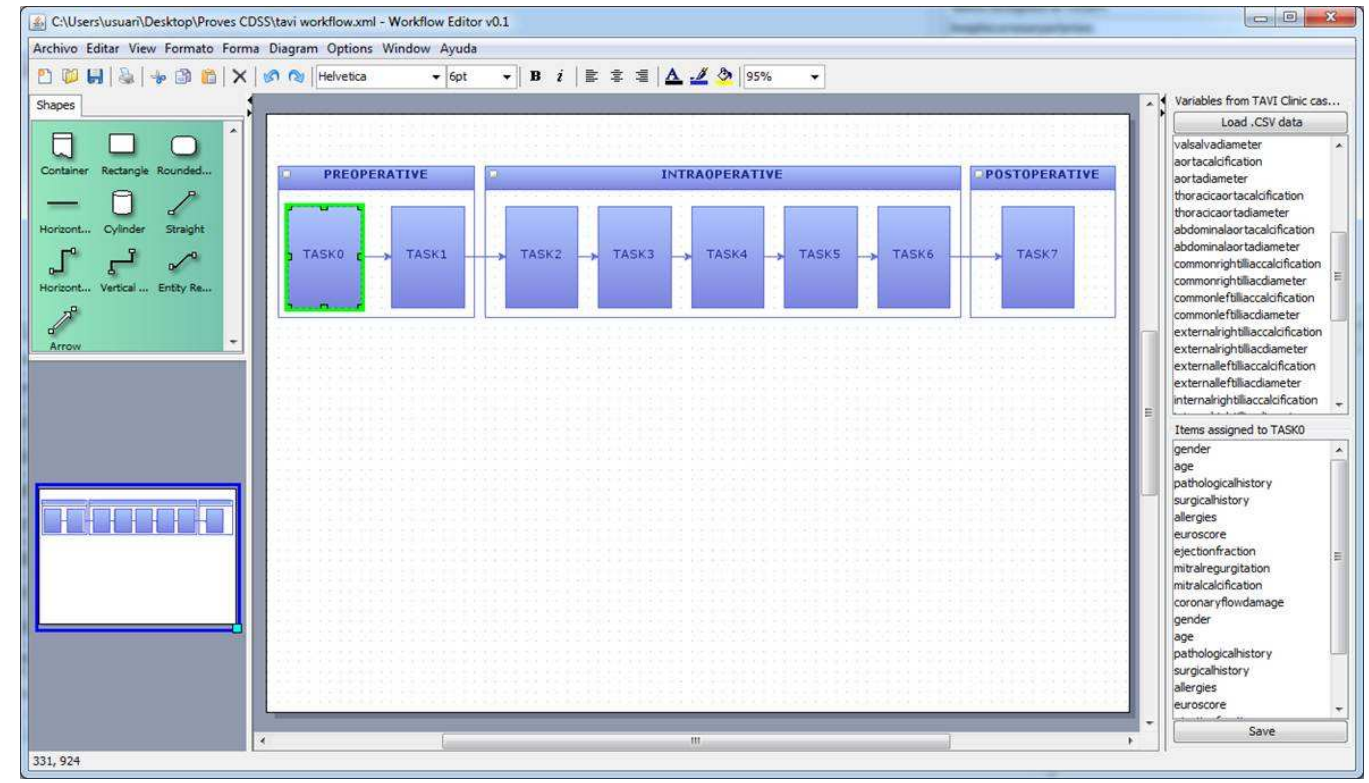

Figure 5: The workflow editor. The interface is accessed through the main application. It offers a complete edition set for workflow management and attributes association.

side of the window, the user proceeds with the attributes-to-task association. By loading the CSV file presented in Section 3.1, the user can access to all the attributes which define a patient's case and associate them to its correspondent task of the workflow. Once the workflow creation is completed, the workflow file is saved as an Extensible Markup Language (XML) file which can be loaded later on by the eXiTCDSS main application in order to proceed with the project development.

\subsection{The interface navigator}

The interface navigator guides the user through all the application possibilities (see Figure 6). The main navigator window contains a table with the case base, the workflow, the attribute/task/phase tree and the features menu. These features have been designed to enhance decision support. The software offers the possibility to load/save different attributes pre-selection. These presets allow clinicians to look for similarities between cases by just working with a specific set of attributes or only considering a single phase of the workflow, for example for carrying out patient studies during the preoperative phase. Other attribute specific selection modes are also included in this feature menu. Repeating experiments and interpreting their results 


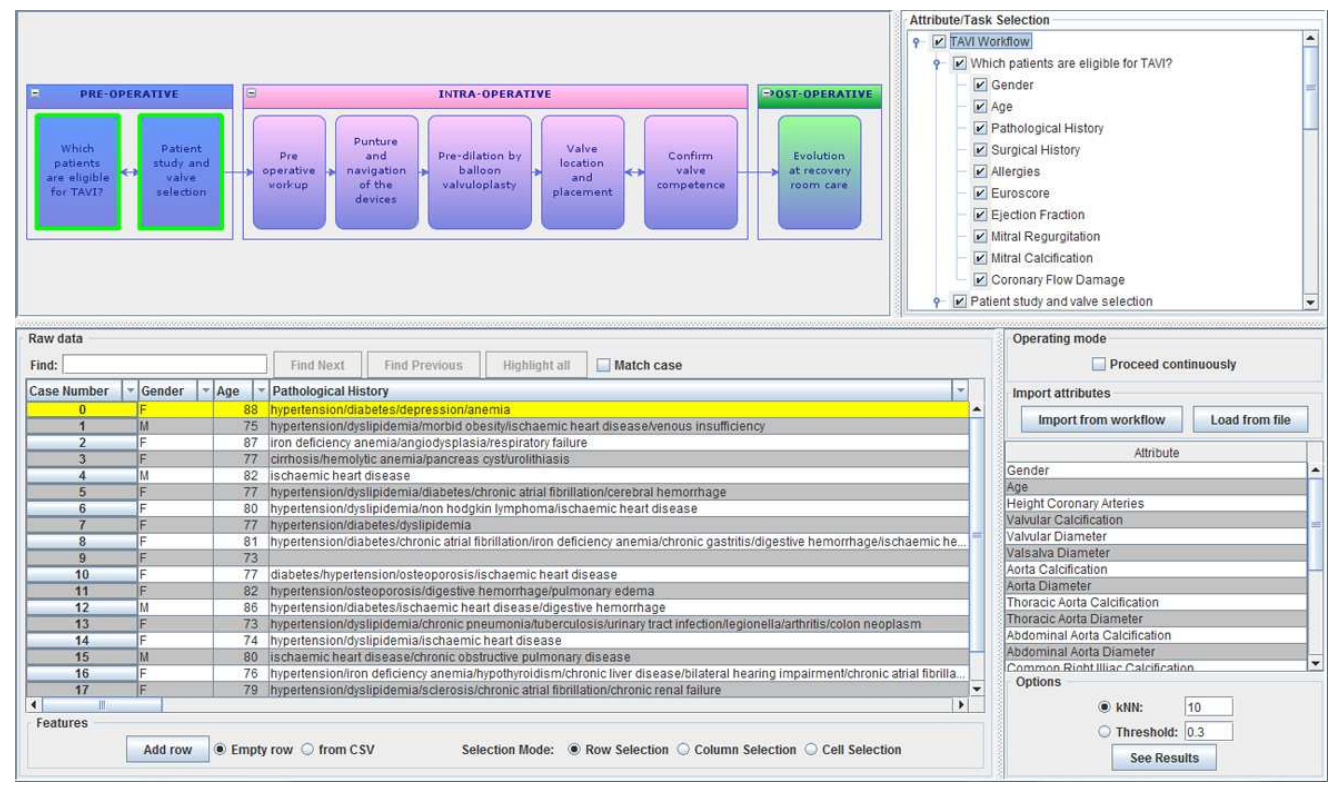

Figure 6: The eXiTCDSS navigator. The loaded workflow is shown on the top of the window. A pull-down tree menu on the top-right of the image allows the user to select/deselect the attributes. Below, the knowledge base is displayed.

are the two key points for clinical support. Reproducibility should guarantee that when the same method and the same test material are used, identical results are obtained. Therefore, all the configuration parameters, distance methods and presets are stored together with the results of every test.

\subsection{The CBR engine}

All the information required to set up a CBR system according to user requirements is stored in the configuration file. The CBR engine is responsible for reading this file, extracting the selected XML and CSV files, methods and parameters and, finally, calling and executing the related CBR algorithms. Therefore, once the configuration file is set, the eXiTCDSS loads the patient case base and the associated workflow file.

\subsubsection{Retrieve module}

The engine compares the stored cases with the current patient data, and selects the most similar cases from the case base. Three key methods are involved in this process. First, the distance method or similarity measure employed to compare cases. In a second place, the methods employed to 
handle missing information when the similarity measures are applied and, finally, the selection procedure to determine the most similar cases. There are local and global similarity measures. Local similarity measures compare two attribute values. There can be as many kinds of local similarity measures as there are operands available. In this work, the local distance selected to handle numeric data is computed by a simple absolute difference of two normalized values, while a discrete distance $d\left(x_{a}, y_{a}\right)$ is set for categorical data. Both methodologies are considered to be commonly used by the community. Local distance methods have to be able to deal with missing values. According to (Zhang et al., 2005), they can be classified as Missing Completely at Random (MCAR), Missing at Random (MAR), and Not Missing at Random (NMAR). MCAR is used when the probability of missing a value is the same for all attributes, MAR is used when the probability of missing a value is only dependent on another attribute, and NMAR is used when the probability of missing a value is also dependent on the value of the missing attribute. Although any of these methods can be implemented and added to eXiTCDSS, for the experimental results presented in this article, we do not distinguish between MCAR, MAR or NMAR values. So, when the value of an attribute either belonging to the query case or to a case of the case base is missing, the attribute is discarded and its local distance does not add towards the global distance computation. When both values are present, the corresponding local distance function is applied. Thus, the local distance concerning attribute $a$ with a value of $x_{a}$ and $y_{a}$ for each case is given by the following equation:

$$
\operatorname{dis}_{L}\left(x_{a}, y_{a}\right)= \begin{cases}\text { discard attribute } & \text { if } x \text { or } y \text { are unknown } \\ d\left(x_{a}, y_{a}\right) & \text { if } x_{a} \text { and } y_{a} \text { are categorical } \\ \left|\hat{x}_{a}-\hat{y}_{a}\right| & \text { if } x_{a} \text { and } y_{a} \text { are numerical }\end{cases}
$$

where

$$
d\left(x_{a}, y_{a}\right)= \begin{cases}1 & \text { if } x_{a} \neq y_{a} \\ 0 & \text { if } x_{a}=y_{a}\end{cases}
$$

Global similarity measures combine different local similarity outcomes to determine the similarity between two cases. The eXiTCDSS framework uses the weighted average (Torra \& Narukawa, 2007), but many others can be 
considered. The global distance is the average of local distances obtained for all the attributes. The global distance between a query case $Q$ and a memory case from the case base $C$, dis $G(C, Q)$, is computed as the Euclidean distance of the previously computed local distances as follows:

$$
\operatorname{dis}_{G}(C, Q)=\sqrt{\frac{\sum_{i=1}^{n} w_{i} d i s_{L}\left(x_{i}, y_{i}\right)^{2}}{W}}
$$

Where $W=\sum_{i=1}^{n} w_{i}, w_{i}$ is the local weight associated to each attribute and defined by the user. Therefore, clinicians can increase or decrease the importance of an attribute by modifying its weight (between 0 and 1) in the CSV file (all weight values set to 1 by default). Finally, $n$ is the amount of attributes considered in the global distance computation.

\subsubsection{Reuse module}

The retrieval phase returns a set of $k$ cases, $C 1, \ldots, C k$, similar to the query case $Q$. The results navigator presents the retrieved cases ranked according to their global distance to $Q$ (i.e. $\operatorname{dis}_{G}(C 1, Q)>\operatorname{dis}_{G}(C 2, Q)>$ $\left.\ldots>d i s_{G}(C k, Q)\right)$. The software allows selecting the $k$ - nearest neighbors or selecting the cases with a distance value smaller than a predefined threshold defined by the user. Then, in the reuse phase the solution to the problem posed by $Q$ should be computed. Particularly, when dealing with a classification problem, the class corresponding to $Q, Q_{\text {class }}$, should be determined. Most of medical CBR systems suggest past solutions without a further adaptation process. The Bilska-Wolak method (Bilska-Wolak \& Floyd, 2002) proposes a probabilistic approach; however, this method has only been applied with a low number of features, and much more research is needed to deploy it in real environments. In this work, the adapted solution $Q^{T}$ is obtained, as shown in Equation 4, by adding the classified solutions of the $r$ retrieved cases, $C_{i}^{T}$, multiplied by the inverse of its global distance measurement.

$$
Q^{T}=\sum_{i=1}^{r} C_{i}^{T} \frac{1}{d i s_{G}(C, Q)}
$$

In this way, the final adapted solution is the result of merging the solutions adopted for the $r$ retrieved past cases, where the most similar solutions have higher impact than the less ones in the final outcome. Note, that we are using 
a very simple case retrieval and reusing methodologies. In this article we do not focus on investigating similarity functions but on the general development of the eXiTCDSS framework and demonstrating its feasibility as a decision support tool for complex clinical procedures.

\subsubsection{Revise module}

Although there are simulators available in other environments (Hammond, 1990), most of the current medical CBR systems rely on human feedback in the revise phase. Once the solutions proposed by retrieved cases have been adapted and executed, the revise step allows users to feedback information into the system and, therefore, to improve the knowledge case base. For the particular case of the eXiTCDSS application, once the results of the executed actions are observed, the module allows clinicians to add revisions, if needed, to the adapted solution $Q^{T}$ of the query case and, if the query case is finally added to the case base at the retain step, the knowledge of the case base will be improved for future querys of similar cases.

\subsubsection{Retain module}

Adding cases automatically to a medical domain without any human control can be somewhat dangerous, since the system can increase the information about a particular case or situation, leading to a large bias in the system. In eXiTCDSS we leave the study and integration of new cases to clinicians. As done in (Schulz, 1999), new cases will be temporary stored in a specific directory until an expert checks them.

\section{4. eXiTCDSS supports Transcatheter Aortic Valve Implantation (TAVI)}

The joint efforts with TAVI professionals from Rennes and Barcelona lead to a specific development of a TAVI intervention workflow (see Figure 7). As can be appreciated, the procedure (from pre-operative to post-operative treatment) is subdivided in 8 different tasks, starting with a study of patient suitability for TAVI in Task 0, to Task 7 where the patient is finally transferred to the Intensive Care Unit (ICU). Parallel to the workflow progress, all the tasks are linked with the different information sources which will provide the necessary attribute data required for defining the patient case profile. Once the workflow and the correspondent attributes associated to

each task have been defined, the user must introduce this information into 


\begin{tabular}{|c|c|c|c|c|c|c|c|c|}
\hline \multirow[b]{2}{*}{$\frac{3}{5}$} & \multicolumn{2}{|c|}{ Pre-operative } & \multicolumn{5}{|c|}{ Intra-operative } & Post-operative \\
\hline & $\begin{array}{c}\text { Task o } \\
\text { Which patients } \\
\text { are eligible for } \\
\text { TAVI? } \\
\text { Contrain }\end{array}$ & $\begin{array}{c}\text { Task } 1 \\
\text { Patient study } \\
\text { and valve } \\
\text { selection } \\
\text { dications }\end{array}$ & $\begin{array}{c}\text { Task 2 } \\
\text { Pre- } \\
\text { operative } \\
\text { workup }\end{array}$ & $\begin{array}{l}\text { Task } 3 \\
\text { Puncture } \\
\text { and } \\
\text { navigation of } \\
\text { the devices }\end{array}$ & $\begin{array}{c}\text { Task } 4 \\
\text { Pre-dilation } \\
\text { by } \\
\text { balloon } \\
\text { valvuloplasty }\end{array}$ & $\begin{array}{c}\text { Task } 5 \\
\text { valve } \\
\text { location and } \\
\text { placement } \\
\text { Re-in }\end{array}$ & $\begin{array}{l}\text { Task } 6 \\
\text { Confirm } \\
\text { valve } \\
\text { competence } \\
\text { flate }\end{array}$ & $\begin{array}{c}\text { Task } 7 \\
\text { Track } \\
\text { evolution at } \\
\text { recovery } \\
\text { room care }\end{array}$ \\
\hline 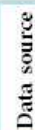 & $\begin{array}{l}\text { TTE/TEE } \\
\text { CT } \\
\text { Fluoroscopy }\end{array}$ & $\begin{array}{c}\text { Angiography } \\
\text { MRI }\end{array}$ & $\begin{array}{c}. \text { Vital } \\
\text { sign } \\
\text { monitor }\end{array}$ & $\begin{array}{c}\text { CT } \\
\text {.Fluoroscopy } \\
\text { Angiography }\end{array}$ & $\begin{array}{l}\text { TTE/TEE } \\
\text { Angiography }\end{array}$ & $\begin{array}{c}\text { TTE/TEE } \\
\text { Angiography } \\
\text { CT }\end{array}$ & $\begin{array}{l}\text { TTE/TEE } \\
\text { Angiography }\end{array}$ & $\begin{array}{c}\text { TTE/TEE } \\
\text { Angiography } \\
\text {.CT }\end{array}$ \\
\hline
\end{tabular}

Figure 7: The workflow depicts all the steps of a TAVI procedure. Together with the main phases and tasks, the data source where attributes are extracted from is also illustrated.

the eXiTCDSS by means of the workflow editor, see Figure 5, presented in Section 3.2. Then, the resultant XML workflow file can be loaded together with the CSV file containing the knowledge base to create the TAVI project inside the main eXiTCDSS window (see Figure 6), the interface navigator, previously described in Section 3.3. From here, the user can start exploring the eXiTCDSS functionalities. The case base table and the attributes list offer numerous possibilities of selection. When dealing with a query case, clinicians can select the tasks or the specific attributes considered the most relevant for obtaining the desired solution. The results offered by the eXiTCDSS application after the retrieve phase are illustrated in the results navigator window illustrated in Figure 8.

The most similar cases to the query case are shown together with its global distance. Also the class corresponding to the query case $Q, Q_{\text {class }}$, is depicted in the console situated at the bottom-right corner of the image. The application allows the user to track the different tasks of the intervention workflow with its associated attributes while consulting the case base. Also, if the medical staff needs additional information for decision making, every case of the case base is linked to its complete clinical history where clinicians can consult any image or file.

\section{Results on the TAVI case base}

The results presented along this section show the application performance when giving support to specific decisions taken during a TAVI procedure. 


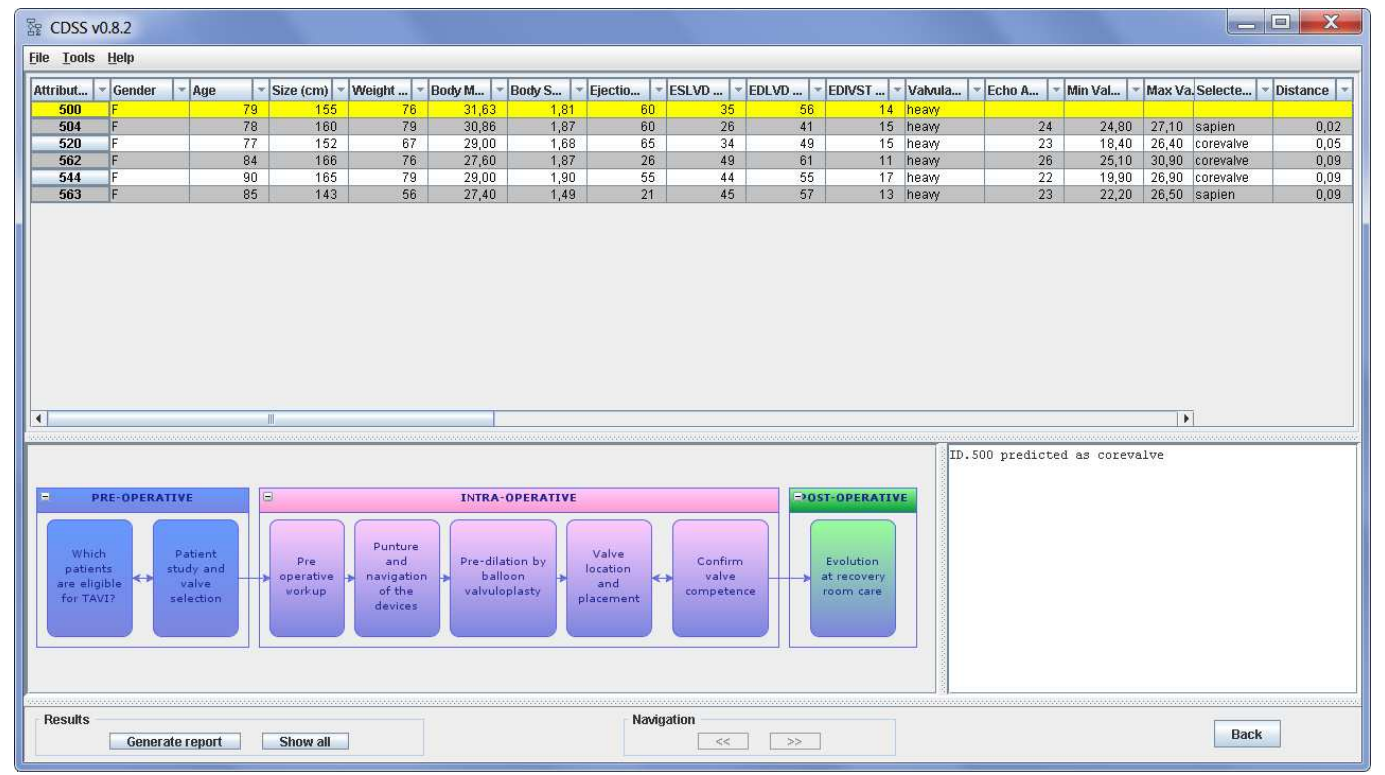

Figure 8: The results navigator window. On the top of the image the query case is presented together with the retrieved cases. The adapted solution for the query is depicted in a console at the bottom-right section of the image.

As commented in the introduction, a TAVI intervention is considered a complex procedure and several critical decisions are taken along the whole process. For demonstrative purposes, the results presented in this article are focused on three critical decisions to take: which Vascular access?, which Valve model? and which Valve size?. Consulted clinicians consider that the success or the failure of the intervention is highly dependant on the right selection of these three attributes. All these decisions are taken by an expert team during the pre-operative phase and specifically at Task 1 - Patient study and valve selection. The vascular access is related with selecting the best way for accessing the aortic valve location. The valve model concerns the decision of choosing the valve type that better fits the patient. Finally, the valve size is related with the selection of the right diameter for the new valve depending on various patient aortic features. The TAVI patient case base used for these experiments has been built with 82 real TAVI patients from the University Hospital of Rennes from which the solution class is known. The eXiTCDSS application shows these results to the user in the results window of the interface navigator detailed in Section 3.3. These results present the confusion matrix, which contains information about the real classification of the cases 
and the prediction given by the system. Also, the results detail information about true positive, true negative, false positive and false negative rates.

\subsection{Attribute selection}

In a TAVI procedure, most of the attributes used in the eXiTCDSS knowledge base are acquired through various imaging system devices such as Ultrasound (US) (Transthoracic Echocardiogram (TTE)/Transesophageal Echocardiogram (TEE)), Fluoroscopy, Angiography, Computed Tomography (CT), Magnetic Resonance Imaging (MRI). Also some data is extracted from the patient historic profile, which contains information about patient pathologies, allergies or past interventions. From the attribute point of view, focusing on the three critical decisions selected for which the eXiTCDSS application is going to show its capabilities, experts agree that not all the attributes from the knowledge base are needed to take these decisions and only some of them are considered directly related with each of them. Therefore, by using one of the eXiTCDSS functionalities presented in the previous section, clinicians can select specific attributes from the case base table and store them into preset files. These files can be modified, add/remove attribute selection, anytime and are stored for future use. Experts have identified an attribute subset for each of the three decisions, Vascular access, Valve model and Valve size, which are considered to contain the most valuable information.

Vascular access. Gender, PLA-like diameter of aortic annulus, valvular calcification, ejection fraction, thoracic aorta calcification, left iliac artery diameter, right iliac artery diameter, subclavian artery diameter, femoral arteries calcification, tortuosity of subclavian artery.

Valve model. Vascular access, PLA-like diameter of aortic annulus, left iliac artery diameter, right iliac artery diameter.

Valve size. Gender, Valve model, PLA-like diameter of aortic annulus, valvular calcification, ejection fraction, thoracic aorta calcification.

Note that the Valve model objective class includes the vascular access and, in the same way, the Valve size contains both, the access and the model. The reason for this is that, according to the clinicians, these decisions are taken following a particular sequence. First they need to know if a TAVI intervention may be feasible by any of the possible accesses. Once the access is granted, in a second step, experts choose the best valve model depending 
on the access selected and the patient's vascular features. Finally, related with the valve type and the vascular features, the clinical team chooses the valve size. The next sections show the results obtained in a cross validation test where the 82 cases act as a query case and the eXiTCDSS application uses the rest of the case base to give the best solution to each decision: vascular access, valve type and valve size.

\begin{tabular}{lcccc}
$\begin{array}{l}\text { Vascular } \\
\text { Access }\end{array}$ & $\begin{array}{c}\text { Trnsfemoral } \\
\text { (predicted) }\end{array}$ & $\begin{array}{c}\text { Subclavian } \\
\text { (predicted) }\end{array}$ & $\begin{array}{c}\text { Trnsapical } \\
\text { (predicted) }\end{array}$ & $\begin{array}{c}\text { Trnsaortic } \\
\text { (predicted) }\end{array}$ \\
\hline Trnsfemoral $_{r}$ & 63 & 0 & 0 & 0 \\
Subclavian $_{r}$ & 6 & 2 & 0 & 0 \\
Trnsapical $_{r}$ & 3 & 1 & 3 & 0 \\
Trnsaortic $_{r}$ & 3 & 0 & 1 & 0 \\
\hline
\end{tabular}

Table 1: Confusion matrix for the vascular access objective class.

\subsection{Vascular access}

As previously mentioned, the vascular access is related with selecting the best way for accessing the aortic valve location. Nowadays, 4 different access points are practiced in a TAVI intervention: transaortic, transapical, subclavian and transfemoral. In a transaortic approach, the valve is inserted on the catheter through an upper ministernotomy or a right anterior minithoracotomy and then through a small hole in the aorta. A transapical procedure, access to the valve location is gained through the left ventricular apex by a left anterolateral minithoracotomy. The subclavian approach uses the subclavian artery to access the valve location. Finally, in a transfemoral approach the valve location is gained through a puncture in the left or the right femoral artery. Table 1 illustrates the confusion matrix for the vascular access objective class. As previously mentioned, the confusion matrix contains information about the real classification of the cases and the prediction given by the system. The columns represent the eXiTCDSS predictions while the real outcomes correspond to the matrix rows. The results show most of the classification errors are produced when trying to give a solution for transaortic, transapical and subclavian approaches. The main reason for these results are the poor representation that these particular approaches have in the knowledge database, most of them being transfemoral approaches. 


\begin{tabular}{lcc} 
Vascular Access & $F P R$ & $T P R$ \\
\hline Trnsfemoral & 0.1463 & 1 \\
Subclavian & 0.0135 & 0.25 \\
Trnsapical & 0.0133 & 0.4286 \\
Trnsaortic & 0.0 & 0.0 \\
\hline Correctly Classified Cases & 68 & $82.9 \%$ \\
Incorrectly Classified Cases & 14 & $17.1 \%$ \\
Total Number of Cases & 82 & \\
\hline
\end{tabular}

Table 2: FPR and TPR and global classification results for the vascular access objective class.

Table 2 shows the total classification percentages for the vascular access objective class. Also, the FPR, which computes the fraction of false positives out of the negative and the TPR, which computes the fraction of true positives out of the positives, for the vascular access objective class are shown. As can be seen, the rates of false positives are satisfactory low despite the small case base used. Regarding the classification results, despite the small case base size, are good enough offering a rate of correct classifications of almost $83 \%$.

\begin{tabular}{lcc}
$\begin{array}{l}\text { Valve } \\
\text { Type }\end{array}$ & $\begin{array}{c}\text { SAPIEN } \\
\text { (predicted) }\end{array}$ & $\begin{array}{c}\text { CoreValve } \\
\text { (predicted) }\end{array}$ \\
\hline SAPIEN $_{r}$ & 35 & 10 \\
CoreValve $_{r}$ & 9 & 28 \\
\hline
\end{tabular}

Table 3: Confusion matrix for the valve type objective class.

\subsection{Valve model}

The valve model concerns the decision of choosing, among the two available options, the valve type that better fits the TAVI patient. As commented in the introduction, there are two current market leaders: the CoreValve and the SAPIEN device. Each one offers different properties and require a particular strategy for their successful placement. Table 3 illustrates the confusion matrix for the vascular access objective class. In the case of deciding the best valve type, the eXiTCDSS mismatches the same amount of cases when deciding the best valves for them, around 10 errors per type. As can be seen, the population of the two valve models is almost equally distributed along 
the knowledge base and this fact gives the system enough diverse information to give the right output, something that did not happen with the previous vascular access classification.

As shown in Table 4 the rate of correct classifications for the valve model decision is about $77 \%$. Also, the FPR, which computes the fraction of false positives out of the negative and the TPR, which computes the fraction of true positives out of the positives, for the valve model objective class are shown.

\begin{tabular}{lcc} 
Valve Type & $F P R$ & $T P R$ \\
\hline SAPIEN & 0.2432 & 0.7777 \\
CoreValve & 0.2222 & 0.7567 \\
\hline Correctly Classified Cases & 63 & $76.8 \%$ \\
Incorrectly Classified Cases & 19 & $23.2 \%$ \\
Total Number of Cases & 82 & \\
\hline
\end{tabular}

Table 4: FPR and TPR and global classification results for the valve type objective class.

\subsection{Valve size}

the valve size is related with the selection of the right diameter for the new valve. Depending on the manufacturer, there are three possible valve sizes for each valve type: $23 / 26 / 29 \mathrm{~mm}$ are the possible diameters if the experts decide to implant a SAPIEN model and $26 / 29 / 31 \mathrm{~mm}$ if the final decision goes for the CoreValve. As illustrated in Table 5, the number of mismatches is, as happened with the vascular access, very low for this objective decision and again most of the errors committed are concentrated in the class with fewer available cases, in this case for the diameter 23. The numbers show again that the methodology is heavily reliant on the size and richness of the knowledge base.

\begin{tabular}{lccc} 
Valve & 23 & 26 & 29 \\
Size & $($ predicted $)$ & $($ predicted $)$ & (predicted) \\
\hline $23_{r}$ & 7 & 6 & 0 \\
$26_{r}$ & 0 & 43 & 3 \\
$29_{r}$ & 0 & 3 & 20 \\
\hline
\end{tabular}

Table 5: Confusion matrix for the valve size objective class. 
Table 6 shows the rate of correct classifications for the valve size decision is about $85 \%$. Also, the FPR, which computes the fraction of false positives out of the negative and the TPR, which computes the fraction of true positives out of the positives, for the valve size objective class are shown. As can be appreciated, the rates of false positives are again quite small despite the lack of cases in such a complex procedure.

\begin{tabular}{lcc} 
Valve Size & $F P R$ & $T P R$ \\
\hline 23 & 0.0 & 0.5385 \\
26 & 0.25 & 0.9349 \\
29 & 0.0508 & 0.8696 \\
\hline Correctly Classified Cases & 70 & $85.4 \%$ \\
Incorrectly Classified Cases & 12 & $14.6 \%$ \\
Total Number of Cases & 82 & \\
\hline
\end{tabular}

Table 6: FPR and TPR and global classification results for the valve size objective class.

The results presented along this section have shown the eXiTCDSS application performance when giving support to specific decisions taken inside a TAVI procedure. In a general term, these first results are satisfactory and demonstrate that the developed tool has good potential capabilities. Now, the platform basics have been built and the addition of improved capabilities will increase its performance. Also, more conclusive results will be obtained when more cases are available or the software is tested will a more complete knowledge base.

\section{Conclusions and future work}

This article presents eXiTCDSS, a workflow-based CDSS designed to give case-specific assessment to clinicians during complex surgery or MISs. The framework facilitates interaction with physicians, which are guided along the application in a user-friendly way. Its workflow structure offers high versatility allowing the clinicians to decide in which steps of the procedure they wish to receive support. The tasks and attributes selection can easily be saved/loaded into independent files for future use. Although it has been designed to give support to a wide range of interventions, the eXiTCDSS has been initially applied to give support to TAVI interventions. The tool has demonstrated its performance giving support to critical questions of a TAVI procedure with good results. Current work is focused with improving 
the user interface and adding more available options for advanced users: choosing among different distance methods and attribute search algorithms. In the same way, we are working in the implementation of a better default distance calculation method that better fits into the kind of data we are managing so non CBR-expert users can obtain better results without dealing with complex testing. Software architecture modifications will provide the application with voice activation and gesture recognition tools. Finally, the feedback received from the numerous physicians consulted has been very positive and the interest showed in this project is encouraging. Clinicians agree that CDSS are of high value specially in recently growing MIS like TAVI where the number of interventions per year is still low compared to common surgery and which also require expert hands due to its complex procedure.

\section{Acknowledgements}

We would like to give our special thanks to the Hospital Clinic of Barcelona, the Centre Hospitalier Universitaire de Rennes and the Laboratoire Traitement du Signal et de l'Image from the University of Rennes for helping us with the case model definition and giving us example cases of TAVI patients. This work has been financed by the Spanish Government Commission Ministerio de Industria, Turismo y Comercio (MITyC) under the project PLAN AVANZA 2 labeled by Information Technology for European Advancement 2 (ITEA2). Also, this research project has been partially funded through the project labeled DPI2011-24929.

\section{References}

Aamodt, A., \& Plaza, E. (1994). Case-based reasoning: Foundational issues, methodological variations, and system approaches. Artificial Intelligence Communications, 7, 39-52.

Baxt, W. (1995). Application of artificial neural networks to clinical medicine. Lancet, 346(8983), 1135-1138.

Bellazi, R., Montani, S., Portinale, L., \& Riva, A. (1999). Integrating rulebased and case- based decision making in diabetic patient management. In 3rd International Conference in Case-Based Reasoning ICCBR'99 (pp. 386-400). Seeon Monastery, Bavaria, Germany. 
Berner, E. (2009). Clinical decision support systems: State of the Art. AHQR Publication No. 09-0069-EF Agency for Healthcare Research and Quality.

Bilska-Wolak, A., \& Floyd, C. (2002). Development and evaluation of a case-based reasoning classifier for prediction of breast biopsy outcome with bi-radstm lexicon. Medical Physics, 29(9), 2090-2100.

Carayon, P., Karsh, B., Cartmill, C., Hoonakker, P., Hundt, A., Krueger, D., \& Thuemling, T. (2010). Incorporating health information technology into workflow redesign. Technical Report 10-0098-EF Center for Quality and Productivity Improvement, University of Wisconsin-Madison.

Coiera, E. (October 2003). The Guide to Health Informatics (2nd Edition). London, UK: Arnold.

Crowley, R., Naus, G., \& Friedman, C. (2003). Development of visual diagnostic expertise in pathology: An information processing study. Journal of American Medical Informatics Association, 10(1), 39-51.

Das, M., \& Eichner, J. (2010). Challenges and Barriers to Clinical Decision Support Design and Implementation Experienced in the Agency for Healthcare Research and Quality CDS Demonstrations. AHQR Publication No. 10-0064-EF Agency for Healthcare Research and Quality.

Demmer-Fushman, D., \& Lin, J. (2007). Answering clinical question with knowledge-based and statistical techniques. In Association for Computational Linguistics (pp. 63-103).

Dombal, F., Leaper, D., Staniland, J., McCann, A., \& Horrocks, J. (1972). Computer-aided diagnosis of acute abdominal pain. British Medical Journal, 2(5804), 9-13.

Dugas, M., Schauer, R., Volk, A., \& Rau, H. (2002). Interactive decision support in hepatic surgery. BMC Medical Informatics and Decision Making, 2.

Durieux, P., Nizard, R., Ravaud, P., Mounier, N., \& Lepage, E. (2000). A clinical decision support system for prevention of venous thromboembolism: effect on physician behavior. Journal of the American Medical Association, 283(21), 2816-21. 
Evans, R., Pestotnik, S., \& Classen, D. (1998). A computer-assisted management program for antibiotics and other anti-infective agents. New England Journal of Medicine, 338, 232-238.

Hammond, K. (1990). Explaining and repairing plans that fail. Artificial Intelligence, 45(1-2), 173-228.

Holst, H., Astrom, K., \& et al., A. J. (2000). Automated interpretation of ventilation-perfusion lung scintigrams for diagnosis of pulmonary embolism using artificial neural networks. European Journal of Nuclear Medicine, 27, 400-406.

Hunt, D., Haynes, R., Hanna, S., \& Smith, K. (1998). Effects of computerbased clinical decision support systems on physician performance and patient outcome: a systematic review. JAMA, 280, 1339-1346.

Laurikkala, J., Juhola, M., Lammi, S., \& Viikki, K. (1999). Comparion of genetic algorithms and other classification methods in the diagnosis of female urinary incontinence. Methods of Information in Medicine, 38, $125-131$.

Ledley, R., \& Lusted, L. (1959). Reasoning foundation of medical diagnosis. Science, 130(3366), 9-21.

Marakas, G. (1999). Decision support systems in the 21st century. Upper Saddle River, New Jersey: Prentice Hall.

Marling, C., \& Whitehouse, P. (2001). Case-based reasoning in the care of alzheimer's disease patients. In 4th International Conference in CaseBased Reasoning ICCBR'01 (pp. 702-715). Vancouver, Canada.

Miller, R., Pople, H. J., \& Myers, J. (1982). Internist-i, an experimental computer-based diagnostic consultant for general internal medicine. New England Journal of Medicine, 307, 468-475.

Niazkhani, Z., Pirnejad, H., Berg, M., \& Aarts, J. (2009). The impact of computerized provider order entry systems on inpatient clinical workflow: A literature review. Journal of the American Medical Informatics Association, 16(4), 539-549. 
Olsson, S., Ohlsson, M., Ohlin, H., \& Edenbrandt, L. (2002). Neural networks, a diagnostic tool in acute myocardial infarction with concomitant left bundle branch block. Clinical Physiology and Functional Imaging, 22, 295-299.

Patel, V., Arocha, J., \& Kaufman, D. (2001). Primer on aspects of cognition for medical informatics. Journal of American Medical Informatics Association, 8, 324-343.

Patel, V., Kushniruk, A., Yang, S., \& Yale, J. (2000). Impact of a computerbased patient record system on data collection,knowledge organisation, and reasoning. Journal of American Medical Informatics Association, 7(6), 569-85.

Perreault, L., \& Metzger, J. (1999). A pragmatic framework for understanding clinical decision support. Journal of the Healthcare Information and Management Systems Society, 13(2), 5-21.

Rinkus, S., Walji, M., Johnson-Throop, K., Malin, J., Turley, J., \& Smith, J. (2004). Human-centerd design of a distributed knowledge management system. Journal of Biomedical Informatics, 38, 4-17.

Schulz, S. (1999). Cbr-works - a state-of-the-art shell for case-based application building. In 7th German Workshop on Case-Based Reasoning (GWCBR) (pp. 3-5). Wurzburg, Germany.

Shortliffe, E. (1976). Computer-Based Medical Consultations: MYCIN. New York: American Elsevier Publishing Co.

Shortliffe, E., Axline, S., Buchanan, B., Merigan, T., \& Cohen, S. (1973). An artificial intelligence program to advise physicians regarding antimicrobial therapy. Computer Biomedical Research, 6(6), 544-560.

Sittig, D., Wright, A., Osheroff, J., Middleton, B., Teich, J., Ash, J., Campbell, E., \& Bates, D. (2008). Grand challenges in clinical decision support. Journal of Biomedical Informatics, 41(2), 387-392.

Torra, V., \& Narukawa, Y. (2007). Modeling decisions: Information fusion and aggregation operators. Berlin: Springer. 
Unertl, K., Novak, L., Johnson, K., \& Lorenzi, N. (2010). Traversing the many paths of workflow research: Developing a conceptual framework of workflow terminology through a systematic literature review. Journal of the American Medical Informatics Association, 17(3), 265-273.

Warner, H. (1979). Computer-assisted medical decision-making. New York: Academic Press.

Watson, I. (2002). Applying knowledge management: Techniques for building organisational memories. In 6th European Conference in Advances in CaseBased Reasoning ECCBR'02 (pp. 6-12). Aberdeen, Scotland, UK.

Webb, J., \& Cribier, A. (2011). Percutaneous transarterial aortic valve implantation: what do we know? European Heart Journal, 32, 140-147.

Wills, A., \& Watson, I. (2004). Building a case-based reasoner for clinical decision support. Lecture Notes in Computer Science, 3157, 554-562.

Wood, B., Locklin, R., Viswanathan, A., Kruecker, J., Haemmerich, D., \& Cebral, J. (2007). Technologies for guidance of radiology ablation in the multimodality interventional suite of the future. Journal of Vascular Interventional Radiology, 18, 9-24.

Wyatt, J., \& Spiegelhalter, D. (1991). Field trials of medical decision-aids: potential problems and solutions. In 15th Symposium on Computer Applications in Medical Care (pp. 3-7). Washington, USA.

Zhang, S., Qin, Z., Ling, C., \& Sheng, S. (2005). Missing is useful: Missing values in cost-sensitive decision trees. IEEE Transactions on Knowledge and Data Engineering, 17(12), 1689-1693. 